\title{
Le libre accès : nouveau paradigme de diffusion des savoirs
}

\author{
Valérie Levasseur ${ }^{\mathrm{a}}$
}

RÉSUMÉ. Le libre accès est un mouvement qui prône l'accès gratuit pour tous aux connaissances et aux résultats issus de la recherche sans barrière d'utilisation. Dans un monde où une grande partie de la recherche est subventionnée par des fonds publics, il s'avère logique et essentiel que les résultats de cette recherche puissent profiter à l'ensemble de la population. Plusieurs voies s'offrent aux auteurs désirant diffuser en libre accès les résultats de leurs recherches et, peu importe la voie empruntée, les avantages sont indéniables, autant pour les utilisateurs que pour les auteurs eux-mêmes. L'avantage majeur du libre accès aux savoirs est qu'il permet une plus grande mise en application des résultats de la recherche et stimule l'innovation.

ABSTRACT. Open access is a movement that promotes free access for all to the knowledge and results of research without permission barriers. In a world where much of the research is publicly-funded, it seems logical and essential that the results of such research benefit the entire population. Several roads are available to authors wishing to make available in open access the results of their research and whatever the road taken, the benefits are undeniable, both for the users and for the authors themselves. The principal advantage of an open access to knowledge is that it allows for greater application of research results and that it stimulates innovation.

\section{Historique et définition}

L'arrivée d'Internet a ouvert la voie à de nouveaux modes de communication, mais aussi à de nouvelles possibilités de diffusion des connaissances. Internet procure désormais aux chercheurs un moyen de mettre, à tout moment, à la disposition de tous, les résultats de leurs recherches. Dès les années 1990, plusieurs initiatives de partage des connaissances émergent avec, par exemple, la création d'arXiv (Ginsparg, 2011), la première archive scientifique structurée permettant la diffusion à tous gratuitement des prépublications dans le domaine de la physique; ou la venue d'un nouveau modèle de revues scientifiques dont le contenu est accessible gratuitement aux lecteurs (Laakso et al., 2011); et aussi l'apparition des premières archives ouvertes institutionnelles et disciplinaires (Hameau, 2008; Joint Information Systems Committee (Jisc), 2018).

De ces initiatives isolées de partage libre des connaissances nait une volonté de mettre en commun et de structurer les efforts afin d'élargir la portée des savoirs partagés. À partir de 2001, différentes initiatives et déclarations permettent aux collectivités de se mobiliser pour remédier à la difficulté d'accès aux résultats de la recherche. La première définition officielle de la notion de libre accès sera établie en 2002, par la «Budapest Open Access Initiative ». Elle vise la littérature scientifique qui est alors définie comme celle que les chercheurs donnent sans attente de rétribution, telle que les articles publiés dans les revues avec comités de lecture ainsi que ceux n'ayant pas encore été évalués (prépublications) (Chan et al., 2002). Dans sa traduction francophone, réalisée par l'Institut de l'information scientifique et technique, cette déclaration définit le libre accès ainsi :

Par «accès libre» à cette littérature, nous entendons sa mise à disposition gratuite sur l'Internet public, permettant à tout un chacun de lire, télécharger, copier, transmettre, imprimer, chercher ou faire un lien vers le texte intégral de ces articles, les disséquer pour les indexer, s'en servir de données pour un logiciel, ou s'en servir à toute autre fin légale, sans barrière financière, légale ou technique autre que celles indissociables de l'accès et l'utilisation d'Internet. La

a Bibliothécaire, M. Sc., Université du Québec à Chicoutimi 
seule contrainte sur la reproduction et la distribution, et le seul rôle du copyright dans ce domaine devrait être de garantir aux auteurs un contrôle sur l'intégrité de leurs travaux et le droit à être correctement reconnus et cités (Institut de l'information scientifique et technique (INISTCNRS), 2004).

Cette déclaration propose ainsi un accès non seulement gratuit à la littérature scientifique, mais aussi un usage sans restriction, sans barrière liée à l'autorisation d'utilisation. L'auteur conserve ainsi le seul droit essentiel d'être reconnu et correctement cité par les utilisateurs de l'information. D'ailleurs, de nos jours, pour les auteurs, la définition de tels droits peut facilement être précisée grâce à de nouvelles licences, comme celle du Creative Commons qui constitue un outil simple et efficace pour consentir au libre accès (Creative Commons).

Enfin, la déclaration de Budapest sera suivie par d'autres déclarations et textes dont la «Berlin declaration on open access to knowledge in the sciences and bumanities", qui élargira le concept de libre accès au-delà de la littérature scientifique en proposant un accès universel à toutes connaissances scientifiques ou culturelles, incluant les données de recherche, les logiciels, les documents multimédias, etc. (Max Planck Society, 2003).

\section{Enjeux}

Par ailleurs, si l'arrivée d'Internet a facilité et rendu techniquement possible la diffusion en libre accès des savoirs, ce mouvement a aussi été amorcé par une volonté de contester et de contrer la marchandisation des connaissances. En effet, l'essor d'un quasimonopole au sein de l'édition scientifique fait en sorte qu'aujourd'hui $50 \%$ des revues savantes sont détenues par cinq grandes maisons d'édition (Larivière, Haustein et Mongeon, 2015). Au cours des 20 dernières années, cette situation a permis à ces éditeurs de procéder à une hausse démesurée des coûts d'abonnement aux revues savantes. Seulement entre 1986 et 2011, le coût d'abonnement à ce type de revues pour les bibliothèques universitaires nordaméricaines a fait un bond de $402 \%$ comparativement au coût d'acquisition des livres qui a augmenté de $71 \%$ (Kyrillidou, 2012). Comme de plus en plus d'universités doivent annuler des abonnements aux revuessavantes en raison du coût trop élevé, cela fait en sorte que de moins en moins de chercheurs ont accès aux résultats de la recherche.
Or, puisqu'une grande partie de la recherche est financée par des fonds publics, il s'avère que le monde scientifique fonctionne selon un modèle dans lequel l'argent des contribuables sert à payer non seulement pour la production de l'information scientifique et son évaluation, par les subventions aux chercheurs, mais aussi à en payer l'accès, par le financement des bibliothèques universitaires. Devant ce paradoxe, plusieurs dirigeants et organismes subventionnaires à travers le monde ont récemment mis en place des mesures visant à promouvoir le libre accès. À titre d'exemple, on peut penser au programme Horizon 2020 de la Commission européenne (2013) ou encore à la politique de libre accès des trois organismes subventionnaires fédéraux du Canada, soit les Instituts de recherche en santé du Canada (IRSC), le Conseil de recherches en sciences naturelles et en génie du Canada (CRSNG) et le Conseil de recherches en sciences humaines du Canada (CRSH) (Gouvernement du Canada, 2015). Ces politiques obligent, entre autres, les chercheurs financés par ces fonds à diffuser en libre accès les publications issues de leurs recherches.

\section{Voies du libre accès}

De nombreuses voies peuvent être empruntées par le chercheur pour diffuser en libre accès ses publications issues de la recherche (Piwowar et al., 2018). Parmi les plus courantes, il y a celle appelée voie verte, qui consiste à déposer une version de l'article, autorisée par l'éditeur de la revue, dans un dépôt institutionnel ou disciplinaire (Harnad, 2007). D'ailleurs, la plupart des universités ont développé de telles archives numériques en libre accès. C'est le cas de l'Université du Québec à Chicoutimi dont le dépôt institutionnel a été lancé en 2011 (https://constellation.uqac.ca/). Cette voie a l'avantage d'être gratuite, autant pour l'auteur que pour le lecteur, et elle est complémentaire à la publication dans une revue traditionnelle à abonnement. L'auteur doit simplement s'assurer de déposer la version autorisée et de respecter les conditions de diffusion en libre accès émises par l'éditeur de la revue. De nos jours, la plupart des éditeurs $(74 \%)$ permettent la diffusion en libre accès, dans un dépôt institutionnel, de la postpublication, c'està-dire de la version corrigée, après évaluation par le comité de lecture et correction, mais sans la mise en page de la revue (SHERPA/RoMEO, 2018). Par 
ailleurs, la majorité des bibliothécaires universitaires peuvent accompagner et conseiller les chercheurs dans ce processus et plusieurs bibliothèques universitaires ont créé des guides en ligne pour informer les chercheurs sur la publication et la diffusion en libre accès (p. ex., https://libguides.uqac.ca/libre-acces/libre-acces-publier).

Une seconde voie très commune de diffusion en libre accès est la voie or ou dorée, qui consiste à publier un article dans une revue savante en libre accès, faisant ainsi en sorte que le lecteur ait accès gratuitement au contenu de la revue (Piwowar et al., 2018). S’il y a quelques années, ces revues étaient mal perçues par les chercheurs, plusieurs d'entre elles ont maintenant établi leur notoriété et certaines ont désormais un facteur d'impact. Par contre, ces revues sont parfois administrées selon le modèle auteur-payeur et dans ce cas, les frais de publication sont transférés à la charge de l'auteur plutôt qu'au lecteur. Bien que parmi les 12152 revues recensées dans le « Directory of Open Access Journal $(D O A J)$ » seulement $26 \%$ imposent des frais de publication aux auteurs, dans de pareils cas, ces frais peuvent s'avérer très élevés. Malheureusement, au cours des dernières années, le monde de l'édition scientifique a vu naitre un lot de nouveaux éditeurs dont l'unique motivation est le potentiel mercantile de ce modèle d'affaires. Souvent qualifiés d'éditeurs prédateurs ou de revues prédatrices, ces revues ne se préoccupent pas de la qualité scientifique et ne répondent pas aux standards de l'édition savante. Les auteurs doivent, par conséquent, être vigilants lors du choix de la revue dans laquelle publier. Il devient primordial d'examiner la crédibilité de la revue en vérifiant, par exemple, le recensement réel de la revue dans les bases de données, le processus de validation et d'évaluation des articles, la crédibilité du comité d'éditorial, etc. Plusieurs guides et listes de vérification existent dans le but d'outiller le chercheur à ce propos (p. ex., https://thinkchecksubmit.org/, http://www.carlabrc.ca/fr/comment-evaluer-une-revue/) et les bibliothécaires ont aussi l'expertise nécessaire pour accompagner le chercheur dans cette démarche.

Enfin, une autre voie communément offerte à l'auteur, et qui peut être considérée comme un dérivé de la voie or, est celle hybride. Ainsi, un éditeur de revue traditionnelle, dont le contenu est normalement accessible par abonnement, offre aux auteurs de diffuser leur article en libre accès sur le site de la revue, à condition de payer des frais de publication souvent très élevés (Piwowar et al., 2018). Dans ce modèle, l'éditeur traditionnel trouve une manière de tirer un profit monétaire supplémentaire des nouvelles exigences en matière de libre accès auxquelles font face les chercheurs et du mode d'évaluation de la recherche qui priorise toujours la publication dans des revues à haut facteur d'impact. Or, il s'avère que ce modèle entraîne souvent une double facturation pour l'accès à la recherche, car non seulement l'auteur paie pour y diffuser son article en libre accès, mais bien souvent il arrive que la revue fasse aussi partie des abonnements déjà payés par la bibliothèque universitaire. Ce modèle permet ainsi aux revues traditionnelles, dont la majorité est détenue par les cinq principaux éditeurs scientifiques, d'engranger des profits toujours plus élevés.

\section{Avantages du libre accès}

Si les voies sont nombreuses pour parvenir à une diffusion libre des savoirs et que certains pièges peuvent surgir, il n'en demeure pas moins que les avantages du libre accès sont indéniables. Cet accès universel aux savoirs profite autant aux auteurs eux-mêmes qu'aux lecteurs et utilisateurs qui deviennent dès lors infinis, allant bien au-delà d'un simple public d'abonnés (Lebert, 2012). Pour l'auteur, cet accroissement de visibilité entraîne nécessairement un taux de citation plus élevé des articles publiés en libre accès par rapport à ceux disponibles uniquement par abonnements (Archambault, Côté, Struck et Voorons, 2016; Hitchcock, 2004; Piwowar et al., 2018). Cette augmentation de la visibilité des chercheurs contribue également à la valorisation de la recherche qui bénéficie, par le fait même, à la réputation de l'université. Enfin, le mouvement de libre accès est aussi étroitement lié à une mission fondamentale de ces institutions soit la diffusion des connaissances et la démocratisation des savoirs (Lebert, 2012).

D'autre part, pour les utilisateurs, lecteurs, enseignants, étudiants et bibliothèques, l'exemption des barrières de prix ouvre un accès plus large aux résultats de la recherche, réduisant ainsi l'écart entre riches et pauvres. Si de nos jours même les universités les mieux nanties peinent à offrir un minimum d'abonnements aux revues savantes pertinentes pour leurs chercheurs, les bénéfices du libre accès prennent tous leurs sens au-delà des murs des universités, soit dans 
les entreprises, les gouvernements, les milieux communautaires, les établissements d'enseignement et les médias. Dans ces milieux, le besoin d'accès aux savoirs est tout aussi présent, mais les difficultés d'y accéder sont encore plus grandes. Le libre accès permet de faire tomber ces barrières, offrant ainsi aux acteurs du milieu la possibilité de mettre à profit et d'utiliser concrètement les résultats de la recherche (Lebert, 2012).

En fait, le libre accès permet d'augmenter la portée et l'efficacité de la recherche, en stimulant l'innovation, par une plus grande accessibilité aux nouvelles connaissances. D'ailleurs, depuis quelques années, ce mouvement tend à s'élargir au-delà des publications et vise maintenant l'atteinte d'une science globalement plus ouverte. À titre d'exemple, les organismes subventionnaires fédéraux canadiens ont entrepris de grandes réflexions et démarches en vue d'élargir le principe de libre accès aux données de recherche proprement dites. Une telle politique, à l'instar de la politique sur le libre accès aux publications issues de la recherche, contribuera à accroitre la visibilité et les retombées de la recherche, mais facilitera également la reproductibilité et la validation des résultats de recherche (Gouvernement du Canada, 2016).
Enfin, le libre accès permet de satisfaire à l'objectif premier de la recherche, qui est de produire de nouvelles connaissances dans le but que celles-ci soient réutilisées, mises en application, ou inspirent d'autres recherches (Harnad et Santiago-Delefosse, 2004).

\section{Outils du libre accès}

Il existe une grande variété d'outils permettant de faciliter la découverte des documents diffusés en libre accès. D'entrée de jeu, les géants comme Google et Google Scholar offrent des solutions populaires. Bien que ces outils soient de puissants moteurs de recherche, ils présentent tout de même certaines faiblesses. Google recense effectivement une masse incroyable d'informations, mais il est difficile d'y limiter les résultats à des documents savants et issus de la recherche, ce que Google Scholar permet aisément, mais avec le désavantage de ne pas permettre de distinguer ce qui est en libre accès de ce qui est disponible uniquement sous abonnement. Ainsi, certains outils spécialisés permettent un recensement plus aisé des documents en libre accès ou en facilitent, du moins, le repérage (tableau 1).

\begin{tabular}{|c|c|}
\hline Outils & Description \\
\hline $\begin{array}{l}\text { Directory of Open Access Journals } \\
\text { (DOAJ) } \\
\text { https://doaj.org/ }\end{array}$ & $\begin{array}{l}\text { Répertoire qui recense plus de } 12000 \text { revues en libre accès } \\
\text { sélectionnées selon des normes de qualité, dont un comité } \\
\text { éditorial et un processus de révision par les pairs. Cet outil } \\
\text { permet aussi de chercher au niveau des articles dans plus de } \\
9000 \text { de ces revues. }\end{array}$ \\
\hline $\begin{array}{l}\text { Bielefeld Academic Search Engine } \\
\text { (BASE) } \\
\text { https://www.base-search.net/ }\end{array}$ & $\begin{array}{l}\text { Moteur de recherche de ressources Web universitaires en } \\
\text { libre accès, exploité par la bibliothèque de Bielefeld Univer- } \\
\text { sity. Cet outil donne accès à plus de } 120 \text { millions de docu- } \\
\text { ments provenant de plus de } 6000 \text { sources académiques. }\end{array}$ \\
\hline $\begin{array}{l}\text { Unpaywall } \\
\text { https://unpaywall.org/ }\end{array}$ & $\begin{array}{l}\text { Outil qui permet de repérer la version en libre accès de plus de } \\
20 \text { millions d'articles académiques provenant d'éditeurs ou de } \\
\text { dépôts institutionnels ou disciplinaires. Cet outil offre la possibi- } \\
\text { lité d'ajouter une extension aux navigateurs Chrome et Firefox } \\
\text { permettant de repérer facilement les versions légalement diffu- } \\
\text { sées en libre accès de publications repérées sur Internet. }\end{array}$ \\
\hline $\begin{array}{l}\text { Kopernio } \\
\text { https://kopernio.com/ }\end{array}$ & $\begin{array}{l}\text { Cet outil est une extension de navigateur qui permet, dans un } \\
\text { premier temps, de faire le lien avec les collections de son uni- } \\
\text { versité d'attache lorsque c'est le cas. Sinon, l'outil permet de } \\
\text { repérer facilement les versions légalement diffusées en libre } \\
\text { accès de publications repérées sur Internet. }\end{array}$ \\
\hline $\begin{array}{l}\text { Connecting Repositories } \\
\text { CORE } \\
\text { https://core.ac.uk/ }\end{array}$ & $\begin{array}{l}\text { Ce moteur de recherche référence au-delà de } 130 \text { millions de } \\
\text { documents provenant de plus de } 10000 \text { revues en libre accès } \\
\text { et d'environ } 3700 \text { dépôts institutionnels et disciplinaires. }\end{array}$ \\
\hline
\end{tabular}

Tableau 1 - Liste alphabétique non exhaustive d'outils permettant le repérage de documents en libre accès 


\section{RÉFÉRENCES}

Archambault, É., Côté, G., Struck, B. et Voorons, M. (2016). Research impact of paywalled versus open access papers. OANumbr, 1(1), 1-5. Repéré à http://digitalcommons.unl.edu/scholcom/29

Chan, L., Cuplinskas, D., Eisen, M., Friend, F., Genova, Y., Guédon, J.-C., ... Velterop, J. (2002). Budapest open access initiative. Repéré à https://www.budapestopenaccessinitiative.org/read

Commission européenne (2013). Horizon 2020. Le libre accès aux publications et aux données de recherche. Repéré à http://www.horizon2020.gouv.fr/cid82025/le-libre-acces-aux-publications-aux-donnees-recherche.html

Creative Commons (2018). Share, collaborate, remix, reuse. Repéré à https://creativecommons.org/

Ginsparg, P. (2011). ArXiv at 20. Nature, 476, 145-147. doi: 10.1038/476145a

Gouvernement du Canada. (2016). Politique des trois organismes sur le libre accès aux publications. Repéré à http://www.science.gc.ca/eic/site/063.nsf/fra/h F6765465.html?OpenDocument

Gouvernement du Canada (2018). Gestion des données de recherche. Repéré à http://www.science.gc.ca/eic/site/063.nsf/fra/h 547652FB.html

Hameau, T. (2008). Les archives ouvertes, un autre mode de publication. Repéré à http://openaccess.inist.fr/?-Archives-ouvertes-

Harnad, S. (2007). The green road to open access: a leveraged transition. Dans G. Anna (dir.), The culture of periodicals from the perspective of the electronic age (p. 99-105). Paris, France : L'Harmattan. Repéré à https://eprints.soton.ac.uk/265753/

Harnad, S. et Santiago-Delefosse, M. (2004). Maximiser l'impact de la recherche en psychologie au moyen de l'auto-archivage. L'initiative pour l'accès libre aux articles scientifiques. Pratiques psychologiques, 10(3), 273-282. doi: 10.1016/j.prps.2004.07.005

Hitchcock, S. (2015). The effect of open access and downloads (hits) on citation impact: A bibliography of studies. Repéré à http://opcit.eprints.org/oacitation-biblio.html

Institut de l'information scientifique et technique (INIST-CNRS) (2004). Initiative de Budapest pour l'accès ouvert. Repéré à http://openaccess.inist.fr/?Initiative-de-Budapest-pour-l

Joint Information Systems Committee (Jisc) (2018). OpenDOAR Statistics. Repéré à http://v2.sherpa.ac.uk/view/ repository visualisations/1.html

Kyrillidou, M. (2012). Research library trends: A historical pictures of services, resources, and spending. Research Library Issues: A Quarterly Report from ARL, CNI, and SPARC, 280, 20-27. Repéré à http://publications.arl.org/rli280/

Laakso, M., Welling, P., Bukvova, H., Nyman, L., Bjork, B. C. et Hedlund, T. (2011). The development of open access journal publishing from 1993 to 2009. PLoS One, 6(6), e20961. doi: 10.1371/journal.pone.0020961

Larivière, V., Haustein, S., et Mongeon, P. (2015). The oligopoly of academic publishers in the digital era. PLoS One, 10(6), e0127502. doi: 10.1371/journal.pone.0127502

Lebert, M. (2016). Présentation de l'accès ouvert [traduction d'un texte de Peter Suber]. Repéré à https://marielebert.wordpress.com/2012/09/11/accesouvert/

Max Planck Society. (2003). Berlin declaration on open access to knowledge in the sciences and humanities. Repéré à https://openaccess.mpg.de/Berlin-Declaration

Piwowar, H., Priem, J., Larivière, V., Alperin, J. P., Matthias, L., Norlander, B., ... Haustein, S. (2018). The state of OA: a large-scale analysis of the prevalence and impact of Open Access articles. Peer,, 6, e4375. doi: 10.7717/peerj.4375

SHERPA/RoMEO. (2018). Publisher copyright policies \& self-archiving. Repéré à http://www.sherpa.ac.uk/romeo/ 